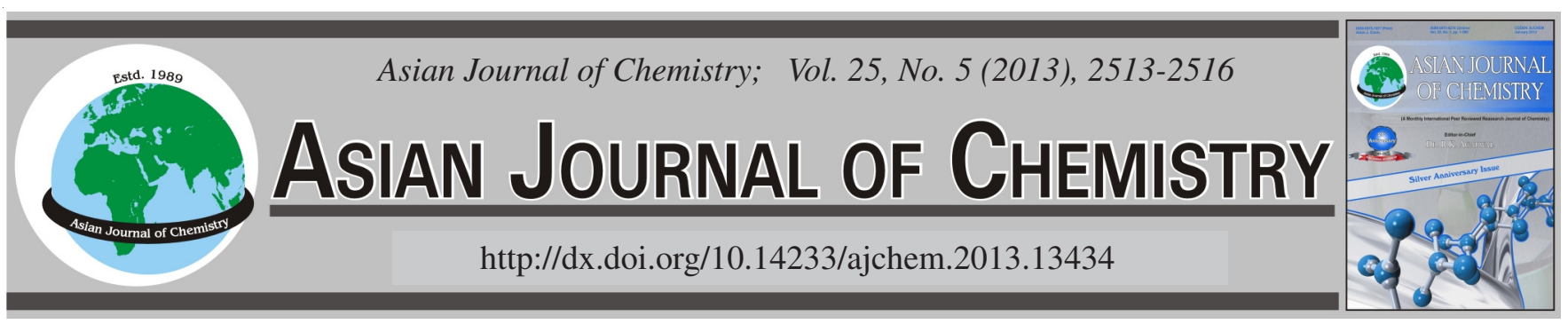

\title{
Water-Dispersible Ru(0) Nanorods With High Aspect Ratio: Microwave Synthesis, Characterization, Formation Mechanism and Catalytic Activity
}

\author{
Shuge Peng ${ }^{*}$, Xinjie Fan, Xiaofei Liu and Jun Zhang
}

Key Laboratory of Polymer and Nanomaterials, College of Chemical Engineering and Pharmacy, Henan University of Science and Technology, 471003, Luoyang, P.R. China

*Corresponding author: Fax: +86 379 64232193; Tel: +86 379 64216956; E-mail: sgpeng@ mail.haust.edu.cn

\begin{abstract}
Water-dispersible $\mathrm{Ru}(0)$ nanorods with high aspect ratio have been successfully prepared by alcohol reduction using microwave heating. For the alcohol reduction, $n$-propanol was used as solvent and reducer and poly(N-vinyl-2-pyrrolidone) were used as stabilizer. The effects of the reactant concentrations on the morphology and size of the $\mathrm{Ru}(0)$ nanorods were discussed. The catalytic activity of waterdispersible $\mathrm{Ru}(0)$ nanorods was also investigated in hydrogen generation from the hydrolysis of sodium borohydride. The structure and morphology of the prepared samples were characterized by transmission electron microscopy, X-ray photoelectron spectrum, Fourier transform infrared spectrum (FT-IR) and elemental analysis (ICP-AES). A possible formation mechanism for the Ru(0) nanorods based on the experimental results was also proposed. The water-dispersible $\mathrm{Ru}(0)$ nanorods provided a lower apparent activation energy $\left(\mathrm{E}_{\mathrm{a}}=\right.$ $33.4 \pm 1 \mathrm{~kJ} / \mathrm{mol})$ than bulk $\mathrm{Ru}(0)$ metal and other reported Ru-based supported catalysts for the hydrolysis of $\mathrm{NaBH}_{4}$.
\end{abstract}

Key Words: Ru(0) nanorods, Microwave heating, Alcohol reduction, Hydrogen generation.

\section{INTRODUCTION}

One-dimensional (1D) metallic nanostructures such as wires, rods, belts and tubes have been the focus of intensive research owing to their unique applications in electronics, photography, information storage, photonics, sensing, imaging, medicine, as well as novel chemical and catalytic properties ${ }^{1}$. As an important member of the group of platinum metals, $\mathrm{Ru}$ nanostructures with well-defined dimensions represent a particular class of interesting nanomaterials to synthesize and study because of their unique and interesting activities as the catalyst ${ }^{2-4}$. In the case of catalysis, it is well-established that the activity of a metal nanocrystal can be enhanced by reducing its size ${ }^{5}$. The selectivity, however, is most sensitive to the packing of atoms on the surface or the exposed faces of a nanocrystal ${ }^{6}$. To utilize efficiently the metal nanocrystals, shape control of Ru nanostructure is the key.

However, much effort has been mainly focused on developing diverse methods to obtain size controllable $\mathrm{Ru}$ nanoparticles. Few researches are reported on the special morphologies of Ru nanostructures. Compared with extensive experimental studies on the synthesis of other noble metals nanorods, such as $\mathrm{Ag}, \mathrm{Au}, \mathrm{Pt}$ and $\mathrm{Pd}^{7,8}$, little work has been made for the synthesis of Ru nanorods. By analogy with their study for Ag or other novel metals, similar anisotropic nanostructures of $\mathrm{Ru}(0)$ should be created. Unfortunately, these methods looks like not to be applied to synthesize of $R u(0)$ nanorods. The main reason might due to the particular physicochemical properties of Ru compare to the other novel metals. So far, the study on the morphology control of $\mathrm{Ru}(0)$ nanoclusters still remains a preparative challenge.

So far, only Harpeness et al. ${ }^{9}$, reported the preparation of rod-like $\mathrm{Ru}(0)$ nanostructure with the low aspect ratio of only 1.6. The $\mathrm{Ru}(0)$ nanorods with high aspect ratio have not been prepared. In the present study, we have attempted to synthesize $\mathrm{Ru}(0)$ nanorods with high aspect ratio by a microwave irradiation method in a one-pot. It was found that the diameter and length of $\mathrm{Ru}(0)$ nanorods could be controlled by changing the reaction concentration. To the best of our knowledge, this is the first synthesis of $\mathrm{Ru}(0)$ nanorods by a microwave irradiation method.

\section{EXPERIMENTAL}

Ruthenium chloride hydrate $\left(\mathrm{RuCl}_{3} \cdot \mathrm{nH}_{2} \mathrm{O}\right.$, Ru content $\geq$ $37 \%$ ) was from Kunming Institute of Novel Metals. Poly(Nvinyl-2-pyrrolidone) $(\mathrm{PVP}, \mathrm{m} . \mathrm{w} .=40,000)$ was obtained from Fluka. All the other reagents in the experiments were analytical pure grade and were used as received.

Preparation of $\mathbf{R u}(\mathbf{0})$ nanorods: The typical preparation of $\mathrm{Ru}(0)$ nanorods as following: $0.8325 \mathrm{~g}$ of poly(N-vinyl-2- 
pyrrolidone) (PVP) $\left(2.5 \times 10^{-2} \mathrm{~mol}\right.$, as monomeric unit $)$ and $0.1961 \mathrm{~g}$ of $\mathrm{RuCl}_{3}\left(2.5 \times 10^{-3} \mathrm{~mol}\right)$ were dissolved by $50 \mathrm{~mL}$ of $n$-propanol in a $250 \mathrm{~mL}$ round-bottomed flask at room temperature under magnetic stirring and finally formed a dark red solution. Then the solution was irradiated to reflux by a modified domestic microwave oven $(800 \mathrm{~W}, 2.45 \mathrm{GHz})$ with a refluxing apparatus. After the mixture was refluxed for a certain period of time, the colour of the solution changed from dark red to deep purple. In all the experiments the microwave over was employed at $100 \%$ power. The resulting product could be collected by centrifugation at $10000 \mathrm{rpm} / \mathrm{min}$ for $5 \mathrm{~min}$ and washed three times using ethanol, then dried $10 \mathrm{~h}$ under vacuum at $50^{\circ} \mathrm{C}$.

The diameter and length of $\mathrm{Ru}(0)$ nanorods could be controlled by changing the reaction concentration. The concentrations of $\mathrm{Ru}^{3+}$ were $0.75 \times 10^{-3}, 2.5 \times 10^{-3}$ and $15 \times 10^{-3} \mathrm{~mol} / \mathrm{L}$ at a fixed $\mathrm{Ru}^{3+} / \mathrm{PVP}$ molar ratio of 10 in our experiments, corresponding to sample 1, 2 and 3, respectively.

Catalytic activity tests: The catalytic activity of PVPstabilized $\mathrm{Ru}(0)$ nanorods was determined by measuring the amount of hydrogen generation from the hydrolysis of sodium borohydride solution using the modified water-displacement method $^{10}$. A three-necked flask containing a Teflon-coated stir bar sodium borohydride was placed on a magnetic stirrer. Then, a graduated glass tube filled with water was connected to the three-necked flask to measure the volume of the hydrogen gas evolved in the reaction. Next, a certain concentration $\mathrm{Ru}(0)$ nanorods colloids were injected rapidly into the three-necked flask by glass syringe. Immediately the catalytic hydrolysis reaction of $\mathrm{NaBH}_{4}$ was triggered. The starting point of the catalytic reaction was defined as the time when the catalyst was added to the flask. The volume of hydrogen evolved was measured by recording the displacement of water level at constant pressure. During the measurement, the solution temperature was measured and carefully controlled within \pm $0.2{ }^{\circ} \mathrm{C}$.

Characterization techniques of $\operatorname{Ru}(0)$ nanorods: Transmission electron microscope (TEM) images were measured on a JEOL JSM-200 CX X-ray photoelectron spectrum (XPS) was obtained on an ESCA LAB 220I-XL photoelectron spectrometer using monochromated $\mathrm{AlK}_{\alpha}$ radiation. All binding energy values were referred to carbon $\left(C_{1 s}=284.6\right.$ $\mathrm{eV}$ ). Infrared spectrum (IR) were carried out on a Thermo Nicolet Fourier Transform Spectrum using a KBr disk method. The content of $\mathrm{Ru}$ in the catalysts was determined on an HK2000 ICP-AES.

\section{RESULTS AND DISCUSSION}

Characterization of $\mathbf{R u}(\mathbf{0})$ nanorods: Fig. 1(a-c) shows TEM photographs of the PVP-stabilized Ru nanoclusters obtained at three different $\mathrm{Ru}^{3+}$ concentrations and a fixed $\mathrm{Ru}^{3+} /$ PVP molar ratio of 10 . At the lowest $\mathrm{Ru}^{3+}$ concentration of $0.75 \times 10^{-3} \mathrm{~mol} / \mathrm{L}$, a spherical particles with diameters of about 10-15 nm were produced [Fig. 1(a)]. When the $\mathrm{Ru}^{3+}$ concentration increased to $2.5 \times 10^{-3} \mathrm{~mol} / \mathrm{L}$ [Fig. 1(b)], it appeared that one-dimensional nanorod structure was obtained. At the $\mathrm{Ru}^{3+}$ concentration of $15 \times 10^{-3} \mathrm{~mol} / \mathrm{L}$ [Fig. 1(c)], larger nanorods with diameters of 20-40 nm and lengths of 100-600
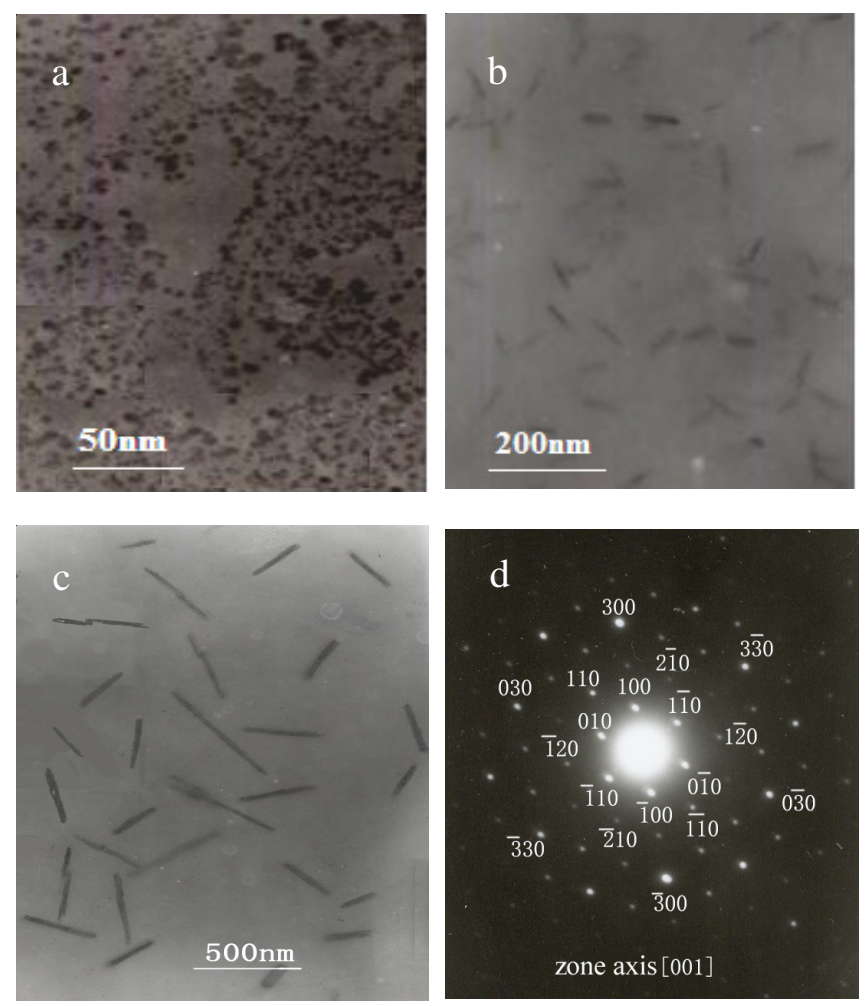

Fig. 1. TEM photographs of $\mathrm{Ru}(0)$ nanorods obtained by microwave heating method. The concentration of $\mathrm{Ru}^{3+}$ were (a) $0.75 \times 10^{-3}$ $\mathrm{mol} / \mathrm{L}$, (b) $2.5 \times 10^{-3} \mathrm{~mol} / \mathrm{L}$ and (c) $15 \times 10^{-3} \mathrm{~mol} / \mathrm{L}$ at a fixed $\mathrm{Ru}^{3+} /$ PVP molar ratio of 10. (d) Electron diffraction pattern of PVPstabilized $\mathrm{Ru}(0)$ nanorods shown in panel (c)

$\mathrm{nm}$ were observed. The aspect ratio of nanorods ranged between 5 and 40. These results indicated that the diameter and length of nanorods were greatly increased with increasing the $\mathrm{Ru}^{3+}$ concentration when $\mathrm{Ru}^{3+} / \mathrm{PVP}$ molar ratio was fixed at 10 . To investigate the crystal structure of nanorods, the electron diffraction pattern was also conducted. A typical result obtained for the nanorods in Fig. 1(c) is shown in Fig. 1 (d). The electron diffraction pattern of nanorods presented the single crystal diffraction design. The measuring d-spacings of (100) and (110) planes were 2.34 and $1.36 \AA$ A, respectively. All the other diffraction spots could be indexed as shown in Fig. 1(d) when the electron beam direction ( zone axis) was [001], which was consistent well with the standard Ru metal data file (JCPDS Card: 06-0663) ${ }^{11}$. In addition, ED pattern of nanorods showed the characteristics of hexagonal compact ( $h$ c $p$ ) structure, which was consistent with the structure of $\mathrm{Ru}$ metal, one of a few transition metals that crystallize only in a hexagonal compact structure.

To further confirm the formation of metallic $\mathrm{Ru}(0)$ nanostructures, XPS measurements were employed to determine the oxidation state of PVP-stabilized Ru nanorods. The results are shown in Fig. 2. The binding energies at 280.0, 483.8 and $461.2 \mathrm{eV}$ in the PVP-coated Ru nanostructures (sample 3 ) were attributed to the binding energy of Ru $3 d_{5 / 2}$, $\mathrm{Ru} 3 \mathrm{p}_{1 / 2}$ and $\mathrm{Ru} 3 \mathrm{p}_{3 / 2}$, respectively, which were consistent with those of Ru metal ${ }^{12}$. Combined with the results of TEM, EDS and XPS, it could be clearly seen that $\mathrm{Ru}(0)$ nanorods had been successfully prepared by alcohol reduction using microwave heating. 

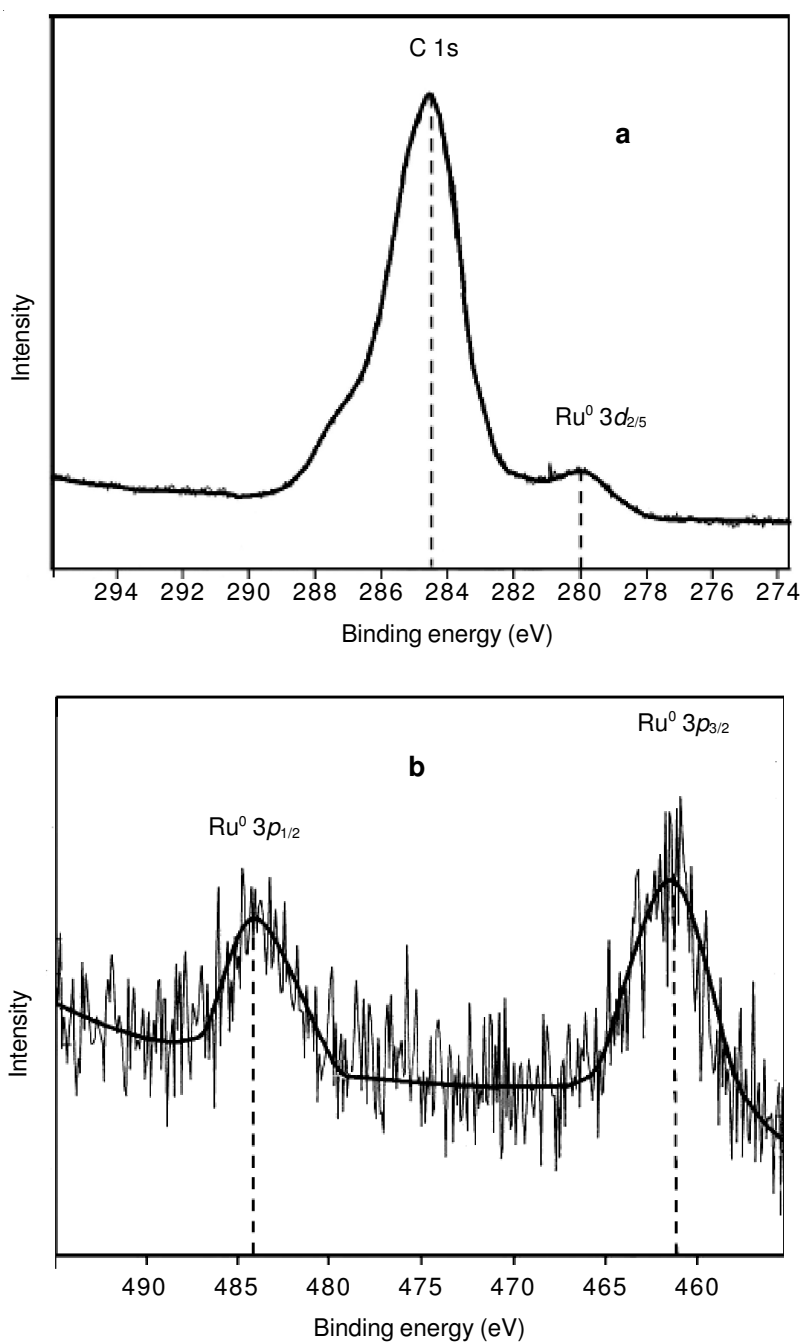

Fig. 2. XPS spectra of PVP-stabilized $\mathrm{Ru}(0)$ nanorods. The four vertical lines indicate the peak positions of the binding energies of $\mathrm{C} 1 \mathrm{~s}$ and $\mathrm{Ru} 3 \mathrm{~d}_{5 / 2}$ (a) and $\mathrm{Ru} 3 \mathrm{p}_{1 / 2}$ and $\mathrm{Ru} 3 \mathrm{p}_{3 / 2}$ (b)

The combination between ruthenium and poly(N-vinyl2-pyrrolidone) was investigated by FT-IR spectroscopic measurement, as shown in Fig. 3. In comparison with the FTIR spectrum of PVP, the absorption band at 2960, 1660, 1280 and $1070 \mathrm{~cm}^{-1}$ were assigned to the vibration models of $\mathrm{C}-\mathrm{H}$, $\mathrm{C}-\mathrm{O}, \mathrm{N}-\mathrm{OH}$ and $\mathrm{C}-\mathrm{N}$, respectively, indicating PVP had been present on the surface of $\mathrm{Ru}$ and served as a capping agent. Furthermore, comparative FT-IR spectra of the PVP-stabilized $\mathrm{Ru}(0)$ nanorods and PVP indicated that the bonding mode of PVP on the nanoclusters surface was due to the carbonyl group coordinated to the $\mathrm{Ru}$ atoms on the nanoclusters surface ${ }^{13}$.

Elemental analysis showed that the Ru content in samples 1,2 and 3 were almost 8.8 wt. \%, close to the initial Ru content in solution: 9.1 wt. \%.

Formation mechanism of $\mathbf{R u}(\mathbf{0})$ nanorods: The formation of anisotropic Ru nanorods probably started by the following reactions according to reduction mechanism of metallic ions in alcohol reduction proposed by Fievet et al. ${ }^{14}$ :

$$
\begin{aligned}
\mathrm{CH}_{3} \mathrm{CH}_{2} \mathrm{CH}_{2}-\mathrm{OH} & \rightarrow \mathrm{CH}_{2} \mathrm{CHO}+\mathrm{H}_{2} \mathrm{O} \\
\mathrm{CH}_{3} \mathrm{CH}_{2} \mathrm{CHO}+\mathrm{Ru}^{3+} \rightarrow \mathrm{Ru}+\mathrm{H}^{+} & \\
+ & \mathrm{CH}_{3} \mathrm{CH}_{2} \mathrm{COCOCH}_{2} \mathrm{CH}_{3}
\end{aligned}
$$

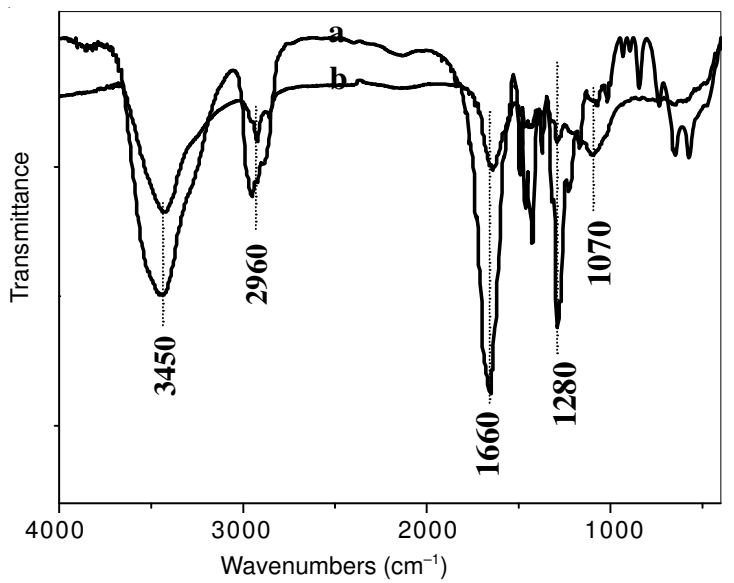

Fig. 3. FT-IR spectra of PVP (a) and PVP-stabilized Ru(0) nanorods (b) prepared by microwave heating method

As a result of the above reduction process, nucleation and growth processes of $\mathrm{Ru}$ nanoparticles obeyed the ostwaldripening theory. Poly(N-vinyl-2-pyrrolidone) acted as a kinetically controller of the growth rates of different crystalline faces through adsorption and desorption. The PVP adsorbed on specific crystalline surfaces could significantly decrease their growth rates and led to a highly anisotropic growth $^{15}$. With the assistance of PVP, some of the large nanoparticles were grown into anisotropic rod-shaped structure. In the oil-bath heating, shuttle-like Ru nanostructres with aspect ratios below 5 were dominantly produced ${ }^{12}$. Generally speaking, microwave heating contributes to the nucleation and growth of metal nanostructures. Ru nanorods under microwave heating must be induced by bursting nucleation due to rapid and homogeneous dielectric heating, which could not be achieved by oil-bath heating.

Catalytic activity: The effect of the temperature on the catalytic activity was investigated by varying temperatures in the range of $10-30{ }^{\circ} \mathrm{C}$ starting with the substrate concentration of $150 \mathrm{mM} \mathrm{NaBH}_{4}$ and catalyst concentration of $0.75 \mathrm{mM}$ $\mathrm{Ru}(0)$ nanorods (sample 3), as shown in Fig. 4. The values of hydrogen generation rate were determined from the linear portions of the $\mathrm{H}_{2}$ volume versus time plots at five different temperatures. As the solution temperature increased, the hydrogen generation rate increased. According to the Arrhenius equation, the relationship of the reaction rate and the activation energy could be expressed as the followings:

$$
\ln \mathrm{r}=-\frac{\mathrm{Ea}}{\mathrm{R}}\left(\frac{1}{\mathrm{~T}}\right)+\ln \mathrm{k}_{0}
$$

where $\mathrm{k}_{0}$ is the pre-exponential parameter, $\mathrm{E}_{\mathrm{a}}$ the activation energy of the reaction, $\mathrm{R}$ the gas constant $\left(8.314 \mathrm{~J} \mathrm{~mol}^{-1} \mathrm{~K}^{-1}\right)$ and $\mathrm{T}$ is the reaction temperature. The inset shows the Arrhenius plot, $\ln \mathrm{r}$ versus the reciprocal absolute temperate $(1 / T)$. According to the slope of the fitted line (-4.02), the apparent activation energy for the hydrolysis of $\mathrm{NaBH}_{4}$ catalyzed by $\mathrm{Ru}(0)$ nanorods could be calculated as $33.4 \pm 1$ $\mathrm{kJ} / \mathrm{mol}$ based on the Arrhenius relationship. Activation energies for the hydrolysis reaction of sodium borohydride solution catalyzed by other Ru-based catalysts are listed in Table- 1 for comparison. Apart from the activation energy found by Özkar and Zahmakiran ${ }^{16}$ with acetate stabilized $\mathrm{Ru}(0)$ nanoclusters, 


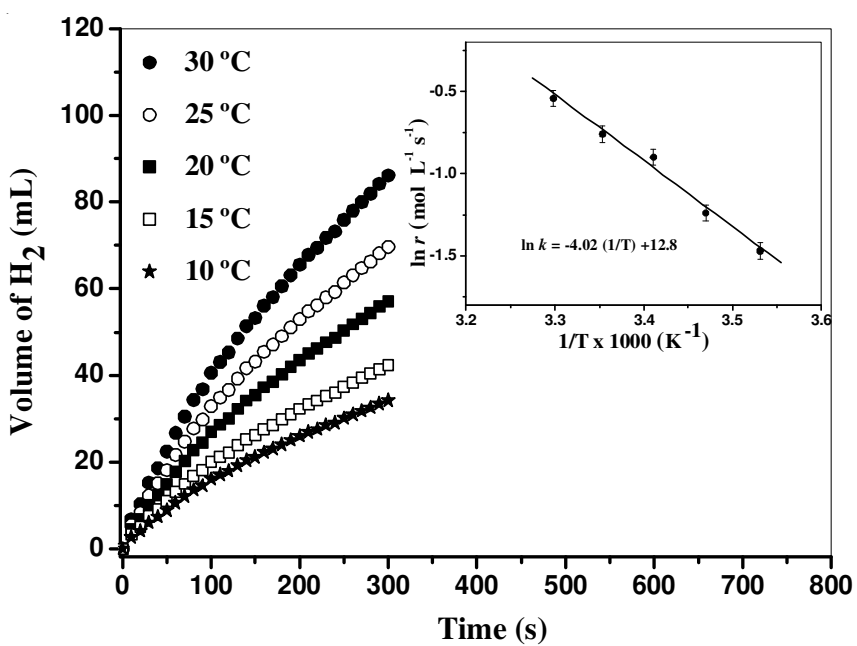

Fig. 4. Volume of hydrogen versus time plot depending on the temperature, and the Arrhenius plot, $\ln \mathrm{r}$ versus the reciprocal absolute temperature $1 / \mathrm{T}$ (the inset) for the hydrolysis of $\mathrm{NaBH}_{4}(150 \mathrm{mM})$ catalyzed by $\mathrm{Ru}(0)$ nanorods at various temperatures in the range of $10-30{ }^{\circ} \mathrm{C}$. The error bars on the plot reflect an around $\pm 5 \%$ measurement error of the hydrogen generation rate

\begin{tabular}{|c|c|c|}
\hline \multicolumn{3}{|c|}{$\begin{array}{c}\text { TABLE-1 } \\
\text { ACTIVATION ENERGY FOR VARIOUS RU-BASED } \\
\text { CATALYST SYSTEMS USED FOR THE } \\
\text { HYDROLYSIS OF SODIUM BOROHYDRIDE }\end{array}$} \\
\hline Catalyst & Activation energy $\left(\mathrm{kJ} \mathrm{mol}^{-1}\right)$ & Reference \\
\hline $\mathrm{Ru}(0)$ nanorods & 33.4 & This work \\
\hline $\mathrm{Ru}(0)$ nanoclusters & 28.5 & 16 \\
\hline $\mathrm{Ru} / \mathrm{LiCoO}_{2}$ & 68.5 & 17 \\
\hline Ru/IRA-400 & 56 & 18 \\
\hline $\mathrm{Ru} / \mathrm{IRA}-120$ & 50 & 19 \\
\hline Ru-promoted sulphated $\mathrm{Zr}$ & 76 & 20 \\
\hline $\mathrm{Ru} / \mathrm{C}$ & 66.9 & 21 \\
\hline Ru/Graphite & 61.1 & 22 \\
\hline
\end{tabular}

the $\mathrm{Ru}(0)$ nanorods stabilized by PVP provided the lowest activation energy for the hydrolysis of sodium borohydride. The high catalytic activities of $\mathrm{Ru}(0)$ nanorods for hydrogen generation from $\mathrm{NaBH}_{4}$ hydrolysis might due to the good dispersion in water and the weak coordination between PVP and $\mathrm{Ru}(0)$ metal.

\section{Conclusion}

In summary, $\mathrm{Ru}(0)$ nanorods stabilized by $\mathrm{PVP}$ with high aspect ratio were obtained in a one pot under microwave irradiation for the first time. The obtained products showed the single crystalline structure of $h c p \mathrm{Ru}(0)$ metal and typical one-dimensional nanorod structure. The diameter and length of nanorods could be varied by changing the concentrations of the $\mathrm{Ru}^{3+}$ and PVP. The kinetics of the hydrolysis of $\mathrm{NaBH}_{4}$ catalyzed by water-dispersible PVP-stabilized $\mathrm{Ru}(0)$ nanorods revealed that $\mathrm{Ru}(0)$ nanorods were highly active catalyst even at room temperatures, providing lower apparent activation energy $\left(E_{a}=33.4 \pm 1 \mathrm{~kJ} / \mathrm{mol}\right)$ than bulk $\mathrm{Ru}(0)$ metal and other reported $\mathrm{Ru}$-based supported catalysts for the hydrolysis of $\mathrm{NaBH}_{4}$.

\section{ACKNOWLEDGEMENTS}

This work was financially supported by National Natural Science Foundation of China (No. 21101058).

\section{REFERENCES}

1. Y. Xia, Y. Xiong, B. Lim and S. E. Skrabalak, Angew. Chem. Int. Ed., 47, 2 (2008).

2. I. Rossetti, F. Mangiarini and L. Forni, Appl. Catal. A, 323, 219 (2007).

3. S. Miao, Z. Liu, B. Han, J. Huang, Z. Sun, J. Zhang and T. Jiang, Angew. Chem. Int. Ed., 45, 266 (2006).

4. C. Roth, N. Benker, R. Theissmann, R.J. Nichols and D.J. Schiffrin, Langmuir, 24, 2191 (2008).

5. C.T. Campbell, S.C. Parker and D.E. Starr, Science, 811, 298 (2002).

6. A. Zecchina, E. Groppo and S. Bordiga, Chem. Eur. J., 13, 2440 (2007).

7. N.R. Jana, L. Gearheart and C.J. Murphy, Chem. Commun., 617 (2001).

8. M.N. Nadagouda and R.S. Varma, Green Chem., 8, 516 (2006).

9. R. Harpeness, Z. Peng, X. Liu, V.G. Pol, Y. Koltypin and A. Gedanken, J. Colloid Interf. Sci., 287, 678 (2005).

10. S. Peng, J. Liu, X. Liu, Y. Zhang and J. Zhang, Adv. Mater. Res., 197198, 1577 (2011).

11. A.N. Grace and K. Pandian, Mater. Chem. Phys., 104, 191 (2007).

12. Y. Zhang, J. Yu, H. Niu and H. Liu, J. Colloid Interf. Sci., 313, 503 (2007).

13. Ö. Metin and S. Özkar, J. Mol. Catal. A: Chem., 295, 39 (2008).

14. F. Fievet, J.P. Lagier, B. Blin, B. Beaudoin and M. Figlarz, Solid State Ionics, 32/33, 198 (1989).

15. M. Tsuji, M. Hashimoto, Y. Nishizawa and T. Tsuji, Mater. Lett., 58, 2326 (2004).

16. S. Özkar and M. Zahmakiran, J. Alloys Comp., 404-406, 728 (2005).

17. Z. Liu, B. Guo, S.H. Chan, E.H. Tang and L. Hong, J. Power Sources, 176, 306 (2008).

18. S.C. Amendola, S.L. Sharp-Goldman, M.S. Janjua, N.C. Spencer, M.T. Kelly, P.J. Petillo and M. Binder, Int. J. Hydrogen Energy, 25, 969 (2000).

19. C.L. Hsueh, C.Y. Chen, J.R. Ku, S.F. Tsai, Y.Y. Hsu, F.H. Tsau and M.S. Jeng, J. Power Sources, 177, 485 (2008).

20. U.B. Demirci and F. Garin, J. Mol. Catal. A, 279, 57 (2005).

21. J.S. Zhang, W.N. Delgass, T.S. Fisher and J.P. Gore, J. Power Sources, 164, 772 (2007).

22. Y. Liang, H.-B. Dai, L.-P. Ma, P. Wang and H.-M. Cheng, Int. J. Hydrogen Energy, 35, 3023 (2010). 